\title{
PROTEOMIC AND OXIDATIVE STRESS ANALYSIS IN HUMAN BRAIN SAMPLES OF HUNTINGTON DISEASE
}

\section{M.Alba Sorolla ${ }^{1}$, Inge Zomer ${ }^{1}$, Gemma Reverter-Branchat ${ }^{1}$, Jordi Tamarit ${ }^{1}$, Isidre Ferrer ${ }^{2}$, Joaquim Ros ${ }^{1}$ and Elisa Cabiscol ${ }^{1}$}

${ }^{1}$ Departament de Ciències Mèdiques Bàsiques, IRBLLEIDA, Universitat de Lleida, Spain; ${ }^{2}$ Institut de Neuropatologia, Servei d'Anatomia Patològica, IDIBELL-Hospital de Bellvitge,

Universitat de Barcelona, Spain

Huntington disease (HD) is an inherited neurodegenerative disorder characterized by degeneration of neurons affecting initially the striatum and progressively the cortex. Clinical symptoms include motor and cognitive alterations leading inevitably to death. The disease involves expansion of CAG trinucleotide repeats in the huntingtin gene codifying for glutamines in the htt protein. We performed a proteomic analysis of human brain post-mortem samples obtained from striatum and cortex of patients with HD compared to samples of age and sex-matched controls. Antioxidant defense proteins that were strongly induced in striatum, but also detectable in cortex, were identified as peroxiredoxins 1, 2 and 6 , as well as glutathione peroxidases 1 and 6 . The activities of other antioxidant enzymes such as mitochondrial superoxide dismutase and catalase were also increased in HD. Aconitase, a protein involved in energy metabolism, showed decreased activities in striatum of HD patients. Protein carbonyls, used as markers of oxidative stress, were increased in HD and glial fibrillary acidic protein was identified as the main target. Moreover, other proteins such us aconitase, $\gamma$-enolase and creatine kinase were also found oxidized in HD. Taken together, these results indicate that oxidative stress and damage to specific macromolecules, would participate in the disease progression from striatum to cortex. Also, these data support the rationale for therapeutic strategies that either potentiate antioxidant defenses or avoid oxidative stress generation in order to delay disease progression. 